\title{
Desigualdade de renda salarial no brasil no período 2001 a 2012
}

\author{
Jair Andrade Araujo ${ }^{1}$ \\ Joyciane Coelho Vasconcelos²
}

Resumo: A alta concentração de renda é um dos principais problemas enfrentados pelo Brasil. Nesse contexto, a importância desse tema se justifica tanto por questões de equidade social quanto pelos efeitos potenciais que a distribuição da renda tem sobre o crescimento econômico, pobreza, bem-estar. Nesse sentido, este trabalho busca mostrar a relação existente entre educação, mercado de trabalho e desigualdade de renda, tendo como base os microdados da PNADs para os anos 2001, 2006 e 2012. Aplicando uma metodologia de decomposição proposto por Fields (2002) foi possível identificar os fatores que causaram a redução na desigualdade de salários no Brasil. Verificou-se que houve discriminação tanto para cor, no ano de 2001 os brancos ganhavam em média $2.51 \%$ a mais que as outras raças. Já em 2012, esse percentual aumentou, os brancos recebiam em média, $4.57 \%$ a mais que os negros, indígenas, parda, e amarela. Também houve uma discriminação para sexo, logo os homens são melhores remunerados que a mulheres, no entanto essa discriminação vem se reduzindo no período analisado. A decomposição mostra que a educação continua sendo a mais importante determinante da desigualdade de renda salarial. Logo, modificações nos níveis educacionais da população brasileiras, possuem uma importância considerável na desigualdade de renda destas, o que deve ser considerado como um fator relevante na consecução de políticas públicas. Palavras Chaves: Desigualdade, Salário, Decomposição.

\begin{abstract}
The high income concentration is one of the main problems faced by Brazil. In this context, the importance of this issue is justified as for social equity as for the potential effects that the income distribution has on the economic growth, poverty and wellness.In this sense, this work aims to show the relationship between education, labor market and income inequality, based on the microdata PNADs for the years 2001, 2006 and 2012. Applying a decomposition methodology proposed by Fields (2002) it was possible to identify the factors that caused the reduction in wage inequality in Brazil.It was found that there was discrimination both for color, in 2001 the whites earned an average of 2,51\% more than other races. In 2012, this percentage increased, whites received an average of $4,57 \%$ more than blacks, Indians, brown and yellow. There was also discrimination for sex, then men are better paid than women, however this discrimination has been reduced during the period. The breakdown shows that education remains the most important

1 Doutor em Economia. Professor do Mestrado em Economia Rural (MAER) da Universidade Federal do Ceará. Pesquisador de Produtividade do CNPq.

2 Economista. Mestre em Economia Rural (MAER) pela Universidade Federal do Ceará. Doutoranda em Desenvolvimento e Meio Ambiente.
\end{abstract}




\section{0 - Desigualdade de renda salarial no Brasil no período 2001 a 2012}

determinant of wage income inequality. Therefore, changes in the educational levels of the Brazilian population, have considerable importance in their income inequality, which should be considered as a relevant factor in achieving public policies.

Keywords: Inequality, Wages, Decomposition.

\section{Introdução}

A má distribuição de renda no Brasil é um dos problemas da economia. Embora continue ocupando um patamar alto, a concentração de renda no País apresenta, nos últimos anos, uma trajetória de queda. Dentro deste contexto de redução da desigualdade de renda, é importante estudar quais fatores que contribuem para diminuição da disparidade de renda brasileira.

Vários estudos documentaram a notável queda da desigualdade de renda ocorrida no Brasil desde o início dos anos 2000. Dentreesses, Barros, Foguel e Ulyssea (2007) fazem um detalhado estudo desse processo. Nesses estudos, a identificação dos determinantes da queda da desigualdade de renda é um dos pontos principais, uma vez que auxilia, por exemplo, a verificação da sustentabilidade desta queda e no desenho de políticas públicas mais eficazes.

Para Berni (2007), no período de janeiro de 1995 a dezembro de 2005, o salário mínimo aumentou $73.15 \%$ em termos reais. Nestemesmoperíodo, a desigualdade de renda da economia brasileira começou a declinar de forma quase ininterrupta. $O$ coeficiente de Gini, que é uma medida de desigualdade, passou de 0.6005, em 1995, para 0.5693, em 2005, ou seja, sofreu uma redução de 5.20\% no período. De acordo com Barros et all. (2007), esta queda, que parece ser pequena, representou uma redução significativa. Para uma amostra de 75 países que possuem dados de evolução da desigualdade na década de noventa, menos de $1 / 4$ apresentou taxas de redução da desigualdade superiores à brasileira.

O mercado de trabalho pode ser um dos responsáveis por esta diminuição na desigualdade de renda por meio dos diferenciais de salários. Dentre esses fatores merecem destaque variáveis de produtividade (educação e experiência), discriminação (raça e gênero) e segmentação no mercado de trabalho (setor produtivo, formalidade, sindicalização, região e dentre outros). Com isso, é possível imaginar que a investigação dos determinantes da renda possa ser fonte para estudar a decomposição das medidas de desigualdade, bem como sua variação entre períodos ou subamostras.

Dada à importância do comportamento do mercado de trabalho na explicação da desigualdade total de renda, deve ser feita uma análise para melhorar o entendimento sobre as causas da desigualdade de renda salarial e de sua queda recente. Uma compreensão das causas da desigualdade é essencial para a escolha de quais políticas públicas devem ser priorizadas.

Este artigo tem como objetivo geral analisar como a educação e outras características 
individuais impactam no salário do indivíduo para Brasil, logodeve-se estimar uma equação de salário ou mincer, decompor os determinantes da desigualdade de renda salarial em uma nova metodologia proposta em Fields (2002). Esta decomposição tem a vantagem de ser possível desagregar seus diferentes componentes segundo a sua contribuição para a desigualdade total. A partir desse método, pode-se obter a contribuição de cada um dos componentes da equação de Mincer na determinação das disparidades salariais.

Objetivos específicos:entender sobre as causas da desigualdade de renda salarial e de sua redução, motivada pela importância do comportamento do mercado de trabalho na explicação da desigualdade total de renda; descrever a evolução da desigualdade de renda do trabalho e da estrutura salarial; e analisar os fatores responsáveis pelas variações na desigualdade de rendimento.

Pretende-se por meio da decomposição responder à seguinte questão: qual é a porcentagem que pode ser atribuída a cada variável explicativa da equação de salários na desigualdade de renda no Brasil?

Para atender ao objetivo proposto nesse trabalho, os dados utilizados foram extraídos da Pesquisa Nacional de Amostragem Domiciliar (PNAD) do Instituto Brasileiro de Geografia e Estatística (IBGE) para os anos de 2001, 2006 e 2012, de forma que possibilitou compreender qual a contribuição de cada fator para a redução de desigualdade.

A equação minceriana que será base para a decomposição do nível e da diferença das medidas de desigualdade incorpora as variáveis: anos de escolaridade, idade, gênero, raça, experiência, ocupação, região, setor de atividade e nível de instrução.

Além da introdução, o presente trabalho está organizado da seguinte forma: a seção dois faz-se uma análise da evolução da desigualdade de renda no Brasil. A terceira seção faz uma revisão da literatura em termos de arcabouços teóricos e empíricos. A quarta é apresentada a metodologia de Fields (2002). A quinta seção faz uma descrição da base de dados. A sexta seção apresenta os resultados e discussões. Em seguida, a sétima seção tem-se os resultados da decomposição em nível e em diferença. Por último, a oitava seção as considerações finais.

\section{Evolução da desigualdade de renda no brasil}

A economia brasileira tem apresentado uma das maiores desigualdades de renda de todo o mundo. Psacharopoulos (1991) mostra que, dentre uma amostra de 56 países, o Brasil apresenta a maior desigualdade de renda. Squire e Zou (1998) mantém o Brasil no topo deste ranking com um coeficiente de Gini médio de 0.578 contra 0.362 do restante da amostra, composta por 49 países, desenvolvidos e em desenvolvimento, para o período de 1947-94.

Os índices de desigualdade da economia brasileira cresceram na década de setenta e se mantiveram elevados até a metade da década de noventa, depois da implementação do Plano Real quando os índices de desigualdade começam a apresentar uma redução.Apesar 
desta queda recente, a desigualdade de renda brasileira permanece ainda bastante elevada. Vale chamar atenção para o fato de que o período de 2001 a 2004 apresentou as menores taxas de desigualdade de renda no Brasil desde a década de sessenta (Kakwani et al., 2006).

A tabela 1, a seguir, mostra a evolução do coeficiente de Gini para a economia brasileira e para as macrorregiões no período de 2001 a 2012. Nota-se que a desigualdade de renda reduziu-se no Brasil, bem como em todas as grandes regiões. Pode-se ver que o coeficiente de Gini para o Brasil caiu de 0.596 para 0.52 no período analisado, que significou uma redução de 0.124 pontos, ou de $12.4 \%$, com um índice superior a 0.50, o país ainda apresenta uma elevada desigualdade. Seguindo a tendência da economia brasileira, todas as macrorregiões também apresentaram uma diminuição na desigualdade de renda. A região Nordeste apresentou uma queda na desigualdade de 0.60 para 0.54, o que representou um decréscimo de 0.105 pontos, ou seja, uma redução de 10.5\%. A Região Sul apresentou para todo o período a menor desigualdade de renda.

Vale ressaltar que nem todas as regiões sofreram uma queda ininterrupta na desigualdade de renda medida pelo Gini no período estudado. As Regiões Sul, Sudeste e Centro-Oeste, nessa ordem, apresentaram as maiores reduções de desigualdade. Em linhas gerais, quando se analisa a variação da desigualdade no período, nota-se que a única região que, desde 2001, apresentou a menor redução na desigualdade foi o Norte, com 10.2\%.

Tabela 01 - Índice de Gini para as Regiões Brasileiras: 2001-2012

\begin{tabular}{c|c|c|c|c|c|c}
\hline Ano & Brasil & Norte & Nordeste & Sudeste & Sul & $\begin{array}{c}\text { Centro- } \\
\text { Oeste }\end{array}$ \\
\hline $\mathbf{2 0 0 1}$ & 0.596 & 0.565 & 0.600 & 0.568 & 0.548 & 0.598 \\
\hline $\mathbf{2 0 0 2}$ & 0.589 & 0.564 & 0.595 & 0.563 & 0.529 & 0.595 \\
\hline $\mathbf{2 0 0 3}$ & 0.583 & 0.542 & 0.585 & 0.557 & 0.531 & 0.581 \\
\hline $\mathbf{2 0 0 4}$ & 0.572 & 0.539 & 0.583 & 0.542 & 0.522 & 0.573 \\
\hline $\mathbf{2 0 0 5}$ & 0.569 & 0.529 & 0.571 & 0.543 & 0.515 & 0.577 \\
\hline $\mathbf{2 0 0 6}$ & 0.563 & 0.521 & 0.573 & 0.538 & 0.506 & 0.563 \\
\hline $\mathbf{2 0 0 7}$ & 0.556 & 0.533 & 0.566 & 0.524 & 0.505 & 0.574 \\
\hline $\mathbf{2 0 0 8}$ & 0.548 & 0.509 & 0.558 & 0.518 & 0.495 & 0.568 \\
\hline $\mathbf{2 0 0 9}$ & 0.543 & 0.523 & 0.558 & 0.511 & 0.491 & 0.560 \\
\hline $\mathbf{2 0 1 1}$ & 0.527 & 0.530 & 0.543 & 0.498 & 0.468 & 0.533 \\
\hline $\mathbf{2 0 1 2}$ & 0.522 & 0.507 & 0.537 & 0.495 & 0.459 & 0.522 \\
\hline $\begin{array}{c}\text { Diferença } \\
(\mathbf{2 0 0 1 - 2 0 1 2 )}\end{array}$ & 0.124 & 0.102 & 0.105 & 0.129 & 0.161 & 0.127 \\
\hline
\end{tabular}

Fonte: Elaboração dos autores a partir dos dados da PNAD. 
Soares (2006) decompõe o coeficiente de Gini e chega à conclusão de que $3 / 4$ da redução na desigualdade de renda no país se deve à diminuição dos diferenciais de salários, enquanto os programas de transferência de renda, do tipo Bolsa Família, são responsáveis pelo $1 / 4$ restante.

\section{Revisão da literatura}

existem vários trabalhos realizados no exterior e no Brasil que tem o objetivo de explicar as causas da desigualdade de renda da economia.

Contreras (2003) determina os principais determinantes da desigualdade de renda no Chile para os anos de 1990 e 1996. Na sua equação de salários, utiliza uma série de variáveis explicativas, tais como anos de escolaridade, experiência, dummies - variável bináriapara gênero e setor de atividade. Os resultados encontrados para a economia chilena mostram que a variável anos de escolaridade explica aproximadamente $20 \%$ da desigualdade naquele país. A segunda variável mais importante foi a dummy - variável binária- utilizada para empregador que mostrou uma contribuição de 7\% para a desigualdade de renda. As contribuições da experiência e do gênero são, respectivamente, 1\% e 2\%.

Recentemente, De Hoyos (2006) faz uma investigação sobre os determinantes da desigualdade de renda no México. Os resultados do seu trabalho mostram que a variável anos de escolaridade é responsável por $24.5 \%$ da desigualdade no ano de 1994. Em 2000, a contribuição desta variável tinha sido de $21.7 \%$. A segunda contribuição mais importante é da variável características do domicílio que representa 7.8\% e 7.4\%, respectivamente, para os anos de 1994 e 2000. A variável experiência não se mostrou importante para explicar a desigualdade de renda na economia mexicana.

Para Lam (1999), que estudou a desigualdade de renda para Korea e África do Sul incluindo três variáveis explanatórias (escolaridade, idade e raça), concluindo que a escolaridade é o principal determinante da escolaridade.

Fields (2002) aplicou a metodologia da decomposição da desigualdade para os Estados Unidos em 1979 e 1999 para identificar os determinantes do aumento da desigualdade dos rendimentos do trabalho que ocorreu no período. Considerou como variáveis explanatórias: gênero, raça, experiência potencial, escolaridade, ocupação, setor e região. A escolaridade exibiu a maior importância relativa para o 'nível' da desigualdade, seguida por ocupação, experiência e gênero. Para a 'diferença' da desigualdade entre 1979 e 1999 , novamente a escolaridade apresentou a maior importância relativa. Conclui que a contribuição da escolaridade para o aumento da desigualdade de renda americana no período foi inteiramente devido ao efeito coeficiente e não por causa do aumento da desigualdade educacional.

Uma das investigações pioneiras que teve como objetivo explicar as causas da desigualdade de renda no Brasil foi o trabalho Langoni (1973), que evidenciou que a variável 


\section{4 - Desigualdade de renda salarial no Brasil no período 2001 a 2012}

educaçãocontribuiu com aproximadamente 58\% do aumento da desigualdade entre as décadas de sessenta e setenta. O autor verificou que, entre as pessoas com nível superior de instrução a contribuição foi de $52 \%$, no fundamental a contribuição foi de $28 \%$, enquanto as pessoas sem instrução não obtiveram nenhuma alteração em seus rendimentos. Para a década de oitenta, Barros e Mendonça (1997) mostram que a educaçãoexplica entre $35 \%$ a $50 \%$ da desigualdade de renda.

Lam e Levinson (1992) também apontam que a variável educaçãotem um peso importante na desigualdade de renda na economia brasileira. De acordo com estes autores, a desigualdade educacional é muito elevada no Brasil, chega a ser seis vezes maior do que nos Estados Unidos, e isto provoca um aumento dos retornos sobre o investimento em educação.

Conforme Barros e Mendonça (1995) a segmentação do mercado de trabalho brasileiro na década de oitenta por ramo de atividade é capaz de promover um diferencial de salários de até 2.4 vezes. Evidenciam que trabalhadores sem carteira assinada ganham de $60 \%$ a $70 \%$ do salário percebido por seus pares com carteira assinada. A variável anos de escolaridade é responsável por 35\% a 50\% da desigualdade de renda no Brasil. Com relação à discriminação por gênero, os autores mostram que os homens, na média, ganham $42 \%$ a mais que as mulheres na década de oitenta.

Segundo Ramos e Vieira (2001) que avaliam a evolução da desigualdade de rendimentos e da estrutura salarial com base nas Pesquisas Nacionais por Amostra de Domicílios (PNADs), para os anos de 1992. Encontraram que a variável escolaridade é responsável por até 30\% da desigualdade quando considerada isoladamente. Concluíram que mudanças associadas ao perfil de rendimentos por grupos educacionais parecem ter sido as principais responsáveis pelo aumento da desigualdade entre 1992 e 1997.

Para Hoffmann (2002) que analisa as distribuições por pessoa economicamente ativa e do rendimento familiar per capita entre 1993 e 2001, utilizando os dados da PNAD, os 5\% mais ricos na população economicamente ativa do Brasil em 2001 são mais de 3.4 milhões de pessoas que ganhavam mais de $\mathrm{R} \$ 3.500,00$ por mês, já considerada a subdeclaração dos rendimentos nas PNADs. Conclui que ocorreu uma redução da desigualdade da distribuição do rendimento por pessoa economicamente ativa, mas que a redução da desigualdade é quase desprezível quando se analisa a distribuição do rendimento familiar per capita.

Na mesma perspectiva, Sachsidaet al. (2004) investigaram o retorno da escolaridade no Brasil e utilizaram os dados da Pesquisa Nacional por Amostra de Domicílio (PNAD) durante o período de 1992 a 1999. Concluíram que o retorno salarial em educação, para 4 anos de estudo, é de 0.1449. Usando esta mesma faixa de educação, mas fazendo uso de uma estimativa por pseudo painel o retorno é de 0.1622 . Em comparação, o resultado obtido por OLS para o mesmo conjunto de dados resultou num retorno em escolaridade de 0.1686 . 
O estudo de Resende e Wyllie (2005) utiliza os dados da Pesquisa sobre Padrão de Vida durante o período de 1996 e 1997, com base em entrevistadas realizadas no Sudeste e no Nordeste do Brasil. Equações de rendimentos para homens e mulheres são consideradas mediante o procedimento para correção do efeito de seletividade amostral concebido por Heckman, incluindo uma medida de qualidade para educação. Conclui que por meio do procedimento de dois estágios de Heckman, as estimativas dos retornos em educação foram de $12.6 \%$ e $15.9 \%$ para mulheres e homens respectivamente.

Hoffmann (2006) também usa a decomposição do coeficiente de Gini para mostrar os determinantes da diminuição da desigualdade de renda brasileira no período de 1997-2004. Seus resultados mostram que aproximadamente 66.5\% desta queda é devido à diminuição dos diferenciais de salários no mercado de trabalho. Os programas de transferência de renda são responsáveis por 28\% desta diminuição.

Na mesma perspectiva Barro et al. (2007), avalia a contribuição expansão educacional para o Brasil, decomporam em dois efeitos, o primeiro devido a reduções no grau de desigualdade educacional e na estrutura etária (efeito quantidade) e o outro devido a reduções na sensibilidade das remunerações a essas duas variáveis (efeito preço). Os resultados obtidos demonstram que um dos principais fatores responsáveis por essa queda da desigualdade de rendimentos do trabalho foi a do efeito preço. Mudanças associadas à escolaridade foram responsáveis por quase $40 \%$ da queda na desigualdade em remuneração do trabalho entre 2001 e 2005, e por quase $20 \%$ da queda na desigualdade em renda familiar per capita.

Recentemente Salvato et al. (2013), aplicaram a metodologia de decomposição de Fields (2002) às equações mincerianas, baseado nas amostragens dos Censos Populacionais brasileiros de 2000 e 2010 do IBGE, apontam que a escolaridade é o principal fator para explicar a desigualdade de renda no Brasil sendo $27.1 \%$ e $19.8 \%$ para os anos, respectivamente.

Salvatoet al. (2003), utilizaram os dados da PNAD e construíram densidades contrafactuaisreponderando a distribuição pelo perfil escolaridade e comparando a Região Sudeste da Nordeste, por meio de uma abordagem não-paramétrica.Os autores concluíram que mais de 50\% de renda é explicado pelo diferencial de escolaridade, a renda do Nordeste reponderada pela escolaridade do Sudeste equivale a 93\% da renda média do Brasil e que o aumento da escolaridade, agrava a desigualdade de renda.

Na opinião de Campanteet al. (2004), que utilizaram a decomposição de OaxacaBlinder ao longo da distribuição de salários na PNAD de 1996 para mensurar o componente de discriminação no diferencial de salários entre brancos e negros, nas regiões Nordeste e Sudeste do Brasil, a discriminação é diferente para as Regiões, sendo de 18\% e $41 \%$, respectivamente.

Já Siqueira e Siqueira (2006) decompôs a desigualdade de renda total na desigualdade de renda no Nordeste intergrupo e intragrupo para o período 1995 - 2004, encontram 


\section{6 - Desigualdade de renda salarial no Brasil no período 2001 a 2012}

que o nível de desigualdade de renda e sua mudança no tempo são causados predominantemente pelo aumento na desigualdade dentro dos estados e não por diferenças na renda média entre eles.

Recentemente, Berni (2007), usa uma abordagem de decomposição para o Nordeste do Brasil mostrou qual é a participação percentual de cada determinante na explicação da desigualdade e a decomposição em diferença que identifica qual é o peso relativo das principais causas da desigualdade da renda dos salários. Para os anos de 1995, 2001 e 2005, também encontra que a escolaridade é variável mais importante para explicação da desigualdade de renda, para os anos de 1995 e 2005, a educação explica respectivamente, $41.82 \%$ e $39.12 \%$ da desigualdade.

Araújo et al. (2008), analisa as áreas rurais do Nordeste do Brasil para os anos 1995, 2001 e 2005. Esses autores apontaram que ocorreu discriminação de cor em alguns estados, mas vem seguindo um ritmo de queda nos últimos anos, pois em 1995, os negros ganhavam em média 21.88\% a menos que os brancos, já em 2005 os negros recebiam em média $12.08 \%$ a menos. Evidenciaram que a variável educação mostrou-se a mais importante na explicação da desigualdade de renda.

Segundo Cruz et all. (2011), é possível identificar por meio do método de decomposição em nível que a variável educação é a mais importante variável na explicação da desigualdade para o período dos anos de 2001, 2004 e 2006, seguida por idade, sexo e indicador de formalidade e sindicalização, destacam ainda que, para o ano de 2001, os anos de escolaridade do trabalhador explicam 10\%, 51.68\%, 28.85\%, 34.11\% e 51.89\% para as regiões do Brasil Norte, Nordeste, Sudeste, Sul e Centro-Oeste, respectivamente. Para os anos de 2004 e 2006, ocorreu uma queda da participação da educação para as regiões Nordeste e Centro-Oeste. Mesmo com essa redução pode-se afirmar que a educação é o fator mais importante na determinação da renda.

No estudo de Suliano e Siqueira (2010) que estima à taxa de retorno da escolaridade da região Nordeste do Brasil: Bahia, Ceará e Pernambuco, observaram que os trabalhadores do setor formal ganham, em média, acima de $12 \%$ que trabalhadores da informalidade.Outro resultado é que o trabalhador sindicalizado chega a ganhar, em média, até 17.79\% a mais que um não sindicalizado no Estado de Pernambuco. Concluíram que a taxa de retorno da escolaridade se encontra acima de 10\%. Quando se considera somente o modelo de variável instrumental, a taxa de retorno da educação para o Estado da Bahia chega a $19.32 \%$ e em Pernambuco a $17.45 \%$.

Segundo Mariano e Arraes (2011) que analisaram o problema da endogeneidade e retornos da educação no Brasil, testaram dois métodos de estimação para a equação minceriana e verificaram que o modelo endógeno apresenta um melhor poder preditivo, optou-se por estimar a equação minceriana por meio da interação entre capital humano e regionalização, visando assim, reduzir o viés de endogeneidade. As taxas de retorno da 
educação variam de acordo com as regiões e estados brasileiros, sendo a região Nordeste e o estado do Ceará os que apresentam o menor retorno, equivalente a, aproximadamente $14.68 \%$, e ao compará-los a região Centro-Oeste e ao estado de Santa Catarina o diferencial entre os retornos chega a $26 \%$ e a $31 \%$, respectivamente. Concluíram que indivíduos advindos de regiões com distinto grau de desenvolvimento econômico não acumulam igualmente o mesmo nível de capital humano.

Pinho Neto et al. (2011), na mesma direção, estuda a evolução e os determinantes da desigualdade de rendimentos do trabalho nas Regiões Nordeste e Sudeste do Brasil, utilizando os dados da PNAD para os anos 2001 e 2008, aplicando a metodologia de decomposição proposta por Fields, encontraram que a educação foi responsável por explicar mais de $40 \%$ da desigualdade de rendimento.

Oliveira e Silveira (2013) também investigam as evidências a respeito das contribuições dos níveis de escolaridade e das mais importantes políticas sociais de transferências de renda para o entendimento não só dos níveis de desigualdade, como também a redução da desigualdade regional de renda observada no Brasil entre os anos de 2003 e 2011. Os resultados obtidos na indicam que, em 2011, cerca de $65 \%$ da desigualdade regional de renda entre os estados brasileiros decorria dos níveis (elevados) de concentração da renda dos indivíduos que possuíam, no mínimo, o ensino médio completo, um percentual superior àquele observado em 2003 (em torno de 58\%), o que decorria de uma distribuição de renda fortemente pró estado rico desta fonte renda.

\section{Metodologia}

\subsection{Decomposição em Nível}

A decomposição em nível tem o objetivo de obter a contribuição de cada variável explicativa da equação Minceriana para a desigualdade salarial. Esta decomposição é originada de uma equação de salários do tipo log-linear:

$$
\ln Y_{i t}=\alpha_{t}^{\prime} Z_{i t}=\sum_{j=1}^{j+z} \alpha_{j t} Z_{i j t}
$$

Em que $\alpha_{t}^{\prime}=\left[\alpha_{t} \beta_{1 t} \beta_{2 t} \ldots \beta_{j t} 1\right]$ e $Z_{t}^{\prime}=\left[1 x_{i 1 t} x_{i 2 t} \ldots x_{i j t} \varepsilon_{i t}\right]$ são vetores- linha de coeficientes e variáveis explanatórias, respectivamente, incluindo o termo de erro aleatório. Dentre as variáveis incluídas na equação estão àquelas associadas à produtividade, discriminação e segmentação no mercado de trabalho.

Conforme detalha Fields (2002) tomando a variância de ambos os lados da equação (1), no lado esquerdo tem-se exatamente uma medida de desigualdade, a log-variância da renda, ou visto sob outro prisma, a covariância de $\operatorname{lnY}$ consigo mesmo, $\sigma^{2}(\ln Y)=\operatorname{cov}\left(\sum_{j=1}^{j+2} \ldots a_{j}, Z_{j}, \ln Y\right)$. Neste sentindo, pode-se escrever a variância incondicional do $\log$ da renda como, 


$$
\sigma^{2}(\ln Y)=\sum_{j=1}^{j+2} \operatorname{cov}\left(a_{j} Z_{j}, \ln Y\right)
$$

Dividindo ambos os lados da equação (2) por $\sigma^{2}(\ln Y)$, pode-se extrair a contribuição de cada fator para a log-variância da renda, $s_{j}(\ln Y)=\operatorname{cov}\left(a_{j}, Z_{j}, \ln Y\right) / \sigma^{2}(\ln Y)$, cuja soma será igual ao coeficiente de determinação da regressão, $R^{2}(\ln Y)$, se eliminar o último elemento de $\mathrm{Z}$ (o erro aleatório),i.e., $\sum_{j=1}^{j+1} s_{j}(\ln Y)=R^{2}(\ln Y)$. Além disso, trata-se de uma decomposição completa, $\sum_{j=1}^{j+2}: \cdots: s_{j}(\ln Y)=100 \%$.

Usando o conceito de correlação, pode-se reescrever a contribuição de cada fator para a log-variância da renda, identificando os efeitos coeficientes, correlação e desvio-padrão.

$$
s_{j}(\ln Y)=\frac{\operatorname{cov}\left(a_{j}, Z_{j}, \ln Y\right.}{\sigma^{2}(\ln Y)}=\frac{a_{j} \sigma\left(z_{j}\right) \operatorname{corr}\left(z_{j}, \ln Y\right)}{\sigma \ln (Y)}
$$

Ou seja, $S_{j}\left(\ln Y_{i}\right)=\frac{\beta_{j}^{*} \sigma\left(x_{i j}\right) * \operatorname{correl}\left(x_{i j}, \ln Y_{i}\right)}{\sigma\left(\ln Y_{i}\right)}$

Onde cada $S_{j}\left(\ln Y_{i}\right)$ representa a parcela da variância do logaritmo da renda, ou seja, da desigualdade, atribuída a j-ésima variável explicativa da equação de salários, incluindo o erro aleatório, que é um substituto das variáveis omitidas.

Por fim, a fração que é explicada por cada fator (variável explanatória) da variância, condicional ao vetor de variáveis explanatórias, $\mathrm{Z}_{-}^{\prime}=\left[1 \mathrm{x}_{1 \mathrm{t}} \mathrm{x}_{2 \mathrm{t}} \ldots \mathrm{x}_{\mathrm{jt}}\right]$, que exclui o termo de erro aleatório, pode ser definida por:

$$
\mathrm{p}_{\mathrm{j}}\left(\frac{\ln \mathrm{Y}}{\mathrm{Z}_{-}}\right)=\frac{\mathrm{s}_{\mathrm{j}}(\ln \mathrm{Y})}{\mathrm{R}^{2}(\ln \mathrm{Y})}
$$

Fields (2002) demonstra que, observados os seis axiomas de Shorrocks (1982) e dada uma função de geração de renda do tipo (1) e uma medida de desigualdade qualquer definida no vetor de logaritmos da renda, $I\left(\ln Y_{1}, \ln Y_{2}, \ldots . \ln Y_{N}\right)$, a decomposição de desigualdade apresentada acima - equações (3) e (4) - é validada desde que I(.) seja contínua, simétrica e para qual a desigualdade em um vetor de renda no qual os indivíduos recebam a mesma renda média seja zero. Inclui nesta classe de medidas de desigualdade o coeficiente de Gini.

Por fim, Fields (2002) mostra que, satisfeitas os axiomas de Shorrocks (1982), não é necessário discutir qual medida de desigualdade usar, uma vez que a contribuição de cada fator é idêntica para todo $j$-ésimo fator para a classe de medidas de desigualdades aplicadas ao logaritmo da renda, assim como a log-variância. Neste sentido, a decomposição descrita nas equações (3) e (4) é única para tais medidas de desigualdade e descreve o que é denomidada de desigualdade de nível. 


\subsection{Decomposição da Diferença}

Diferentemente da decomposição em nível vista anteriormente, a decomposição da diferença tem o objetivo de atribuir pesos às variáveis que contribuíram para a mudança da desigualdade de renda entre três períodos distintos, no caso desse trabalho 2001, 2006 e 2012. Desta maneira, este segundo tipo de decomposição pode informar quais as variáveis mais relevantes para explicar a diminuição ou o aumento de um determinado índice de desigualdade escolhido.

É possível decompor a variação de um índice de desigualdade entre grupos, países ou tempo conforme a variação de cada um de seus determinantes, chamada de contabilidade da 'diferença' da desigualdade. Segundo Fields (2002) para qualquer medida I(.), calculada para dois grupos (ou períodos) e satisfeitas as condições acima, pode-se decompor sua variação:

$$
\mathrm{I}(.)_{2}-\mathrm{I}(.)_{1}=\sum_{\mathrm{j}}\left[\mathrm{s}_{\mathrm{j}, 2} \mathrm{I}(.)-\mathrm{s}_{\mathrm{j}, 1} \mathrm{I}(.)\right]
$$

Em que $s_{j, k}$, é o peso relativo do fator $j$ no grupo $k=1,2$ (ou período). Neste caso, pode-se escrever a contribuição do fator $j$ na variação da medida de desigualdade I(.). Nesse trabalho o índice de Gini foi escolhido para fazer a decomposição, por ser amplamente utilizado em trabalhos envolvendo distribuição de renda e também pelo fato desse indicador atender a diversas propriedades desejáveis que um indicador deve atender. Então, seguindo Fields (2002), tem-se que:

$$
\Pi_{j}(I(.))=\frac{S_{j, 2} * I(.)_{2}-S_{j, 1} * I(.)_{1}}{I(.)_{2}-I(.)_{1}}
$$

Assim, a decomposição em nível pode ser obtida a partir dos coeficientes estimados na equação de salários, da dispersão, ou desvio padrão, das variáveis utilizadas no modelo e da correlação das respectivas variáveis explicativas com a variável dependente. Logo, para o cálculo dos $S_{j}$ 's será necessário, além dos resultados da regressão, o uso das informações contidas na matriz de correlação e na estatística descritiva dos dados.

Fields (2002) discute ainda como interpretar o resultado de cada fator explanatório dependendo de como eles estão incorporados na equação de determinação da renda. Se os fatores são representados por uma única variável contínua ou discreta (ex.: uma dummy de sindicalização), então a construção da contribuição do fator é direta seguindo as equações (3) a (6). Contudo, três casos deverão ser considerados de forma diferenciada: $i$ ) variáveiscategóricas representadas por um conjunto de dummies; ii) variável explanatória representando efeitos não-lineares; $i i i)$ interação de variáveis explanatórias. Para os dois primeiros casos a solução proposta é a mesma: somar os efeitos. Para o terceiro caso não há uma solução direta e sugere-se reestimar a equação de determinação da renda para os subgrupos destacados na interação. 


\section{0 - Desigualdade de renda salarial no Brasil no período 2001 a 2012}

Por fim, cabe analisar as fontes de contribuição dos fatores para a mudança da desigualdade, decompondo a variação da importância relativa de cada fator, . Sabe-se que a decomposição da diferença em qualquer fator pode ser aproximada pela diferença do logaritmo da equação (3), i.e.

$$
\begin{aligned}
& \frac{\Delta \mathrm{s}_{\mathrm{j}}(\ln \mathrm{Y})}{\mathrm{s}_{\mathrm{j}}(\ln \mathrm{Y})} \approx \frac{\Delta \mathrm{a}_{\mathrm{j}}}{\mathrm{a}_{\mathrm{j}}}+\frac{\Delta \sigma\left(\mathrm{z}_{\mathrm{j}}\right)}{\sigma\left(\mathrm{z}_{\mathrm{j}}\right)}+\frac{\Delta \operatorname{corr}\left(\mathrm{z}_{\mathrm{j}}, \ln \mathrm{Y}\right)}{\operatorname{corr}\left(\mathrm{z}_{\mathrm{j}}, \ln \mathrm{Y}\right)}-\frac{\Delta \sigma(\ln \mathrm{Y})}{\sigma(\ln \mathrm{Y})} \\
& \Delta \% \mathrm{~s}_{\mathrm{j}}(\ln \mathrm{Y}) \approx \Delta \%\left(\mathrm{a}_{\mathrm{j}}\right)+\Delta \% \sigma\left(\mathrm{z}_{\mathrm{j}}\right)+\Delta \% \operatorname{corr}\left(\mathrm{z}_{\mathrm{j}}, \ln \mathrm{Y}\right)-\Delta \% \sigma(\ln \mathrm{Y})
\end{aligned}
$$

Neste sentido, a decomposição da mudança da importância do fator, ,pode ser escrita por:

$$
1 \approx \frac{\Delta \mathrm{a}_{\mathrm{j}}}{\mathrm{a}_{\mathrm{j}}}+\frac{\Delta \sigma\left(\mathrm{z}_{\mathrm{j}}\right)}{\sigma\left(\mathrm{z}_{\mathrm{j}}\right)}+\frac{\Delta \operatorname{corr}\left(\mathrm{z}_{\mathrm{j}}, \ln \mathrm{Y}\right)}{\operatorname{corr}\left(\mathrm{z}_{\mathrm{j}}, \ln \mathrm{Y}\right)}-\frac{\Delta \sigma(\ln \mathrm{Y})}{\sigma(\ln \mathrm{Y})}
$$

O primeiro termo do lado direito da equação (8) representa o efeito coeficiente, ou seja, a mudança na importância do fator devido a mudança do retorno do fator sobre a determinação do log da renda. O segundo termo representa o efeito desvio-padrão do fator, ou seja, a mudança na importância do fator devido à mudança de dispersão do fator. $\mathrm{O}$ terceiro termo representa o efeito correlação, ou seja, a mudança na importância do fator devido a mudança na correlação entre o fator e o log da renda. Por fim, deve-se descontar da mudança da importância do fator aquela devido à própria mudança no log da renda.

Fields (2002) aponta que os primeiro e terceiro componentes são função de $\operatorname{cov}\left(Z_{j}, \ln Y\right)$ e portanto não são ortogonais. Se o objetivo é ter uma decomposição ortogonal, então é necessário decompor a primeira parte da equação (3), em que $s_{j}(\ln Y)=a_{j}^{2} \sigma^{2}\left(z_{j}\right) / \sigma^{2}(\ln Y)$, de modo que se pode fazer a aproximação da decomposição da mudança da importância do fator, $\mathrm{s}_{\mathrm{j}}$, por

$$
1 \approx 2 \frac{\Delta \% \mathrm{a}_{\mathrm{j}}}{\Delta \% \mathrm{~s}_{\mathrm{j}}(\ln \mathrm{Y})}+2 \frac{\Delta \% \sigma\left(\mathrm{z}_{\mathrm{j}}\right)}{\Delta \% \mathrm{~s}_{\mathrm{j}}(\ln \mathrm{Y})}-2 \frac{\Delta \% \sigma(\ln \mathrm{Y})}{\Delta \% \mathrm{~s}_{\mathrm{j}}(\ln \mathrm{Y})}
$$

Na decomposição da diferença, assim como na decomposição em nível, a contribuição, ou peso relativo de cada um dos determinantes da queda/aumento da desigualdade somados deve ser igual à unidade, com a diferença que estes podem ser positivos ou negativos, logo:

$$
100 \%=\sum_{j} \frac{S_{j, 2} * I()_{2}-S_{j, 1} * I(.)_{1}}{I(.)_{2}-I(.)_{1}}=\sum_{j} \Pi_{j}(I(.))
$$

Quando $\Pi_{j}(I(.))_{>0}$, indica que o j-ésimo componente da equação de salários contribuiu para uma queda da desigualdade, caso $\Pi_{j}(I())>$.0 , percebe-se o contrário, esse componente fez com que a desigualdade se elevasse. 
É importante notar que, ao contrário da decomposição em nível, a decomposição da diferença é sensível ao índice escolhido, pois, pode-se perceber que $\Pi_{j}(I())>$.0 é uma função de , ou seja, a contribuição do j-ésimo fator explicativo da renda para a decomposição da diferença depende do índice de desigualdade que está se utilizando.

\section{Base de dados}

Os dados utilizados nesse trabalho foram extraídos da Pesquisa Nacional por Amostra de Domicílios (PNAD) do Instituto Brasileiro de Geografia e Estatística (IBGE). As estimativas serão geradas a partir dos períodos, 2001, 2006 e 2012.

A equação de determinantes do rendimento - equação minceriana - que será base para a decomposição do 'nível' e da 'diferença' das medidas de desigualdade incorpora as variáveis de educação e experiência potencial como fatores de produtividade. Observa-se no quadro 01, a seguir, a descrição das variáveis explicativas da equação de salários.

\section{Quadro 01 - Descrição das Variáveis Explicativas da Equação de Salários}

\begin{tabular}{|c|c|}
\hline Variável & Descrição \\
\hline Educação & $\begin{array}{l}\text { Assume valores de } 0 \text { a } 15 . \text { O valor nulo mostra que o indivíduo não completou o } \\
\text { primeiro ano de escolaridade. Os valores de } 1 \text { a } 14 \text { mostram o número de anos de } \\
\text { educação completos do indivíduo. O valor } 15 \text { significa que o indivíduo possui } 15 \text { ou } \\
\text { mais anos de escolaridade. }\end{array}$ \\
\hline Gênero & Variável binária que assume valor unitário para mulheres e nulo para homens. \\
\hline Exp & Experiência potencial (Idade - anos de estudo). \\
\hline Exp2 & Experiência potencial ao quadrado. \\
\hline Raça & $\begin{array}{l}\text { Variável binária que toma valor unitário para trabalhadores de raça branca e valor } \\
\text { nulo em caso contrário. }\end{array}$ \\
\hline Idade & $\begin{array}{c}\text { Varia de } 18 \text { a } 59 \text { de acordo com os anos completos de idade do indivíduo. Para } \\
\text { mostrar que o logaritmo natural dos salários não varia linearmente à idade, a variá- } \\
\text { vel idade } 2 \text { também é utilizada. }\end{array}$ \\
\hline Sind & $\begin{array}{l}\text { Variável binária que representa a filiação do trabalhador a alguma entidade sindical. Assu- } \\
\text { me valor unitário quando o trabalhador é sindicalizado e valor nulo em caso contrário. }\end{array}$ \\
\hline $\begin{array}{l}\text { Ocupa- } \\
\text { ção }\end{array}$ & $\begin{array}{l}\text { Dummiesde ocupação (empregados sem carteira de trabalho assinada, conta pró- } \\
\text { pria, empregadores) e empregados com carteira de trabalho assinada é a base de } \\
\text { comparação. Por possuírem uma dinâmica salarial diferente, os servidores públicos, } \\
\text { civis ou militares, foram excluídos da amostra. }\end{array}$ \\
\hline $\begin{array}{l}\text { Setor de } \\
\text { atividade }\end{array}$ & $\begin{array}{l}\text { Dummiesde setor de atividade (indústria de transformação, construção civil, co- } \\
\text { mércio e serviços, administração pública, educação saúde e cultura, serviços do- } \\
\text { mésticos) e agricultura é à base de comparação. }\end{array}$ \\
\hline Região & Nordeste, sudeste, sul e centro-oeste; sendo norte a base comparação. \\
\hline
\end{tabular}

Fonte: Elaboração dos autores. 


\section{2 - Desigualdade de renda salarial no Brasil no período 2001 a 2012}

A equação de salários é representada da seguinte forma:

$$
\begin{array}{r}
\ln Y_{i}=\beta_{1}+\beta_{2} E D U_{i}+\beta_{3} S E X O_{i}+\beta_{4} E X P_{i}+\beta_{5} E X P_{i}^{2}+\beta_{6} R A C A_{i}+\beta_{7} \operatorname{SIND}_{i} \\
+\quad \sum_{k=1}^{3} \partial_{k} \operatorname{OCUP}_{k, i}+\sum_{l=1}^{6} \theta_{l} \operatorname{ATIV}_{L, i}+\sum_{p=1}^{4} \pi_{p} R E G_{P, i}
\end{array}
$$

Em que: $\mathrm{H}$ = Logaritmo da renda do trabalho principal; EDUi= Anos de estudo; SEXOi = Dummy de Gênero (Feminino $=1)$; EXPi= Experiência potencial (Idade - anos de escolaridade - 6); EXP2i = Experiência potencial ao quadrado; $\mathrm{RAÇAi}=$ Dummie que toma valor unitário para trabalhadores de raça branca e valor nulo em caso contrário;SINDi= Dummies que assume valor unitário quando o trabalhador é sindicalizado e valor nulo em caso contrário; OCUPk, i = Dummies de ocupação (Empregados sem carteira de trabalho assinada, Conta própria, Empregadores); ATIVl, i= Dummies de setor de atividade (indústria de transformação, construção civil, comércio e serviços, administração pública, educação saúde e cultura, serviços domésticos); e REGp, i= Dummies de região (nordeste, sudeste, sul e centro-oeste).

A tabela 2, a seguir, apresenta as estatísticas descritivas das variáveis explicativas da equação para os anos 2001, 2006 e 2012. Pode-se observar que o trabalhador médio de 2001, 2006 e 2012 possui respectivamente 6.68, 7.46 e 8.39 anos de escolaridade. Isto significa um acréscimo de aproximadamente um ano e meio a mais de educação do trabalhador durante o período 2001-2012. 
Jair Andrade Araujo, Joyciane Coelho Vasconcelos - 173

Tabela 2- Estatísticas Descritivas - Brasil, 2001, 2006 e 2012.

\begin{tabular}{|c|c|c|c|c|c|c|c|}
\hline \multirow{3}{*}{$\begin{array}{c}\text { Grupo da } \\
\text { Variável }\end{array}$} & \multirow{3}{*}{$\begin{array}{c}\begin{array}{c}\text { Variável Indepen- } \\
\text { dente }\end{array} \\
\text { Sexo }\end{array}$} & \multirow{2}{*}{\multicolumn{2}{|c|}{$\begin{array}{c}2001 \\
\text { Desvio- } \\
\text { Média padrão }\end{array}$}} & \multirow{2}{*}{\multicolumn{2}{|c|}{$\begin{array}{c}2006 \\
\text { Desvio- } \\
\text { Média padrão }\end{array}$}} & \multirow{2}{*}{\multicolumn{2}{|c|}{$\begin{array}{c}2012 \\
\text { Desvio } \\
\text { Média padrão }\end{array}$}} \\
\hline & & & & & & & \\
\hline & & 0.40 & 0.49 & 0.40 & 0.49 & 0.41 & 0.49 \\
\hline Raça & Raça & 0.54 & 0.50 & 0.50 & 0.50 & 0.46 & 0.50 \\
\hline \multirow[b]{2}{*}{ Experiência } & Experiência & 28.19 & 12.56 & 28.08 & 12.87 & 28.04 & 12.88 \\
\hline & $\begin{array}{l}\text { Experiência ao } \\
\text { quadrado }\end{array}$ & 952.32 & 780.56 & 954.36 & 790.76 & 952.16 & 786.67 \\
\hline Escolaridade & Educação & 6.68 & 4.24 & 7.46 & 4.25 & 8.39 & 4.15 \\
\hline \multirow{4}{*}{ Ocupação } & $\begin{array}{l}\text { Empregado com } \\
\text { carteira de trabalho } \\
\text { assinada }\end{array}$ & 0.39 & 0.49 & 0.42 & 0.49 & 0.49 & 0.50 \\
\hline & $\begin{array}{c}\text { Empregado sem } \\
\text { carteira de trabalho } \\
\text { assinada }\end{array}$ & 0.30 & 0.46 & 0.29 & 0.45 & 0.25 & 0.43 \\
\hline & Conta Própria & 0.26 & 0.44 & 0.24 & 0.43 & 0.22 & 0.41 \\
\hline & Empregador & 0.05 & 0.22 & 0.05 & 0.22 & 0.04 & 0.19 \\
\hline \multirow{7}{*}{ Atividade } & Agrícola & 0.15 & 0.35 & 0.16 & 0.37 & 0.13 & 0.34 \\
\hline & $\begin{array}{c}\text { Indústria de } \\
\text { Transformação }\end{array}$ & 0.17 & 0.38 & 0.22 & 0.41 & 0.21 & 0.41 \\
\hline & Construção & 0.09 & 0.29 & 0.10 & 0.30 & 0.14 & 0.35 \\
\hline & Comércio e Reparação & 0.19 & 0.39 & 0.27 & 0.44 & 0.27 & 0.45 \\
\hline & Administração Pública & 0.03 & 0.16 & 0.03 & 0.17 & 0.03 & 0.18 \\
\hline & $\begin{array}{c}\text { Educação, Saúde e } \\
\text { Social }\end{array}$ & 0.10 & 0.30 & 0.10 & 0.30 & 0.10 & 0.30 \\
\hline & Serviços Domésticos & 0.27 & 0.45 & 0.12 & 0.32 & 0.11 & 0.31 \\
\hline \multirow{5}{*}{ Região } & Norte & 0.05 & 0.22 & 0.075 & 0.26 & 0.08 & 0.27 \\
\hline & Nordeste & 0.25 & 0.43 & 0.25 & 0.43 & 0.25 & 0.43 \\
\hline & Sudeste & 0.45 & 0.50 & 0.44 & 0.50 & 0.43 & 0.49 \\
\hline & Sul & 0.17 & 0.37 & 0.16 & 0.37 & 0.16 & 0.37 \\
\hline & Centro-Oeste & 0.08 & 0.27 & 0.07 & 0.26 & 0.08 & 0.27 \\
\hline
\end{tabular}

Fonte: Elaboração dos autores a partir dos dados da PNAD.

Nota-se que a proporção de mulheres, em média, aumentou, passando de 0.40 para 0.41, de 2001 para 2012, respectivamente, mostrando que a participação da mulher no mercado de trabalho está aumentando no Brasil. 


\section{4 - Desigualdade de renda salarial no Brasil no período 2001 a 2012}

De 2001 e 2012, verifica-se queem média a proporção de brancos diminui de 0.54 para 0.46, respectivamente; há umaredução do percentual de pessoas com experiência; expansão do percentual de pessoas destinadas ao nível de ocupação Empregados com carteira de trabalho assinada, denotando o aumento da formalização no país, passando de 0.39 para 0.49, em 2001 e 2012, respectivamente.

No grupo de variável atividade, verifica-se que se mantiveram constante no período analisado as atividades administração pública e educação, saúde e social; nota-se que ocorreu uma redução na atividade serviços domésticos, passando de 0.27 para 0.11, 2001 e 2012, respectivamente. Ocorreu em média um aumento na atividade comércio e reparação, de 0.19 para 0.27, em 2001 e 2012, respectivamente.

\section{Método}

Se a equação de salários seguir todas as hipóteses clássicas de regressão sua estimação pode ser feita por mínimos quadrados ordinários, mas se forem violadas é importante usar alguma estratégia econométrica para resolver ou atenuar possíveis problemas. Problemas que podem surgir na estimação da equação de Mincer é a endogeneidade e de causalidade.

Primeiramente estima-se a equação de salários ou equação de Mincer para a decomposição dos determinantes da desigualdade de renda. Um problema é a endogeneidade, isto ocorre quando a correlação entre alguma variável explicativa e o erro aleatório é diferente de zero.Wooldridge (2002) relaciona as três principais fontes de endogeneidade: omissão de variáveis, erros de medição e simultaneidade. No caso da equação de salários explorada neste trabalho, a variável educação é possivelmente endógena.

Existe uma forte correlação entre a educação do indivíduo e seu nível de habilidade, o que pode fazer com que a variável educação torne-se endógena se a habilidade do indivíduo não é observada (CARD.1993). Sachidaet al. (2004) seguem a recomendação de Wooldridge (2002) e tentam solucionar este tipo de problema por meio de uma estimação com mínimos quadrados ordinários de dois estágios com a utilização de variáveis instrumentais.

De acordo com Stock e Watson (2004) a causalidade que geralmente vai da educação para salários também vai dos salários para a educação, na medida em que o nível salarial do indivíduo pode muito bem determinar seu nível ótimo de escolaridade, de forma que ambas as variáveis passam a ser determinadas dentro do modelo. Se for esse o caso, a causalidade vai para trás (de salário para educação), e para frente (de educação para salário), isto é, há causalidade simultânea. Se ela existe, uma regressão por MQO capta ambos os efeitos, de modo que o estimador torna-se viesado e inconsistente.

Para solucionar esse problema, a equação de salário foi estimada e utiliza-se como instrumento a variável número de pessoas por família, também extraída das PNAD’s de 2001, 
2006 e 2012. A ideia para a utilização deste instrumento é que existe uma relação inversa entre educação e número de pessoas por família, como é explicado no estudo de De La Croix e Doepke (2003) e Berni (2007). De acordo com estes autores, quanto maior o número de indivíduos em uma família, menor será os anos de estudos devido a restrição orçamentária e portanto, essa é uma excelente variável que pode ser utilizada como instrumento.

Após a escolha da variável candidata a instrumento é necessária à utilização do teste de Hausman. Este tipo de teste é utilizado para confirmar a endogeneidade da variável educação. Se a hipótese nula de exogeneidade não for aceita, significa que a variável educação é realmente endógena.

O teste de Hausman indicou que a hipótese de exogeneidade foi rejeitada, indicando que a variável educação é endógena na equação de salários, uma vez que, os p-valoresencontramseiguais a zero. Assim, o uso do método de mínimos quadrados de dois estágios (2SLS) é o mais apropriado se comparado aos mínimos quadrados ordinários.

\section{Resultados e discussão}

A tabela 3, a seguir, apresenta as estimativas em segundo estágio obtidas por meio de mínimos quadrados de dois estágios para as equações de salários dos anos de 2001, 2006 e 2012. Todos os coeficientes são estatisticamente significantes ao nível de 1\% e apresentam os sinais esperados.

Os resultados obtidos para os coeficientes da variável educação, os retornos médios da escolaridade foram de 22.32\%, 20.66\% e 16.66\% para os anos 2001, 2006 e 2012, respectivamente. Com isso, já se pode notar que, apesar de ser a variável mais importante para explicar o salário, a educação vem apresentando diminuição de seu retorno médio. De certa forma, esses resultados foram encontrados pordiversos autores, tais como Sachidaet al. (2004) que usa dados da PNAD de 1996, e o modelo estimação de Heckman para corrigir o viés de seleção amostral, encontra-se um retorno médio para os anos de escolaridade de 12.9\% no Brasil. Cruz et all. (2011), estima uma equação de salários para a Macrorregiões do Brasil e encontra para o Nordeste os retornos médios de escolaridade que foram de 21.03\%, 18.18\% e 12.81\%, para os anos de 2001, 2004 e 2006, respectivamente.

Com relação a variável sexo, pode-se verificar que os homens são melhores remunerados que as mulheres, reforça que há discriminação de gênero em favor dos homens no mercado de trabalho brasileiro, mas que o diferencial de rendimentos reduziu, os resultados mostram que as mulheres ganhavam, respectivamente, $51.42 \%$ e $45.12 \%$ a menos que os homens em 2001 e 2012. Esta tendência de queda da discriminação por sexo no mercado de trabalho nordestino também foi verificado por Berni (2007) que mostra que os homens ganhavam em média 51.49\% a mais que as mulheres em 1995 e 35.23\% em 2005.

O retorno da experiência potencial é positivo e reduz com o aumento da experiência (concavidade para baixo da função de rendimentos em relação à experiência, controlado 
pelos demais fatores). Nota-se que a experiência se reduziu de 7,61\% para 3.93\%, de 2001 e 2012, respectivamente.

Para a variável raça, verifica-se que existe discriminação de cor. Por exemplo, no ano de 2001 os brancos ganhavam em média 2.51\% a mais que as outras raças. Esta discriminação segue em queda durante o período analisado, pois em 2006 os brancos recebiam em média 1.56\% a mais que pessoas de outras raças. Já em 2012, esse percentual aumentou, os brancos recebiam em média, 4.57\% a mais que os negros, indígenas, parda, e amarela. Logo, as outras raças possuem em média rendimentos inferiores aos brancos.

Comparando com os trabalhadores com carteira de trabalho assinada, apenas os empregadores possuem rendimento médio superior em 2001, 2006 e 2012, controlado pelas demais variáveis. Contudo, este diferencial aumentou no período de $31.71 \%$ para $50.79 \%$.

Por sua vez, o setor de atividade 'agricultura' possui o menor nível de remuneração ao ser comparado com o setor Construção para os períodos estudados. Administração pública para os anos 2001 e 2006 foi menor remunerado que o setor agrícola, mas em 2012 passa a ser melhor remunerado.

Já Educação, saúde e social e serviços domésticos possuem um rendimento inferior que a atividade agrícola para os anos 2001, 2006 e 2012. Salvatoet al. (2013) mostra que atividade agricultura apresenta o menor nível de renumeração ao ser comparado com os demais setores.

Tabela 3 - Estimativas de Segundo Estágio Brasil, 2001, 2006 e 2012. Variável dependente: $\log$ (rendimento trabalho principal)

\begin{tabular}{|c|c|c|c|c|}
\hline $\begin{array}{c}\text { Grupo de } \\
\text { Variável }\end{array}$ & Variáveis Explicativas & 2001 & 2006 & 2012 \\
\hline \multirow{2}{*}{ Escolaridade } & \multirow{2}{*}{ Educação } & 0.2232 & 0.2066 & 0.1666 \\
\hline & & $(540.01)$ & $(561.80)$ & $(595.51)$ \\
\hline \multirow{2}{*}{ Gênero } & \multirow{2}{*}{ Sexo } & -0.5142 & -0.5181 & -0.4512 \\
\hline & & $(-1814.16)$ & $(-1551.21)$ & $(-1660.90)$ \\
\hline \multirow{4}{*}{ Experiência } & \multirow{2}{*}{ Experiência potencial } & 0.0761 & 0.0668 & 0.0393 \\
\hline & & $(865.04)$ & $(1052.23)$ & $(1131.70)$ \\
\hline & \multirow{2}{*}{$\begin{array}{l}\text { Experiência potencial } \\
\text { ao quadrado }\end{array}$} & -0.0006 & -0.0005 & -0.0002 \\
\hline & & $(-941.89)$ & $(-842.27)$ & $(-258.47)$ \\
\hline \multirow{2}{*}{ Raça } & \multirow{2}{*}{ Raça } & 0.0251 & 0.0155 & 0.0457 \\
\hline & & $(51.21)$ & $(37.86)$ & $(147.71)$ \\
\hline \multirow{2}{*}{ Sindicato } & \multirow{2}{*}{ Sind } & 0.0073 & 0.0144 & 0.0183 \\
\hline & & (13.38) & $(35.14)$ & $(55.46)$ \\
\hline
\end{tabular}


Jair Andrade Araujo, Joyciane Coelho Vasconcelos - 177

Tabela 3 - Estimativas de Segundo Estágio Brasil, 2001, 2006 e 2012.

Variável dependente: log (rendimento trabalho principal) (continuação)

\begin{tabular}{|c|c|c|c|c|}
\hline \multirow{6}{*}{ Ocupação } & \multirow{2}{*}{$\begin{array}{c}\text { Empregado sem carteira de } \\
\text { trabalho assinada }\end{array}$} & -0.2047 & -0.2602 & -0.2384 \\
\hline & & $(-480.64)$ & $(-680.45)$ & $(-735.64)$ \\
\hline & \multirow{2}{*}{ Conta Própria } & -0.2916 & -0.4253 & -0.2823 \\
\hline & & $(-825.90)$ & $(-1279.19)$ & $(-909.95)$ \\
\hline & \multirow{2}{*}{ Empregador } & 0.3168 & 0.3580 & 0.4604 \\
\hline & & $(249.59)$ & $(351.86)$ & $(575.17)$ \\
\hline \multirow{12}{*}{ Atividade } & \multirow{2}{*}{ Indústria de Transformação } & 0.0451 & -0.0718 & 0.0652 \\
\hline & & $(40.14)$ & $(-69.63)$ & $(81.43)$ \\
\hline & \multirow{2}{*}{ Construção } & 0.2148 & 0.1154 & 0.2882 \\
\hline & & $(299.34)$ & $(162.35)$ & $(531.84)$ \\
\hline & \multirow{2}{*}{ Comércio e Reparação } & -0.0112 & -0.0730 & 0.0505 \\
\hline & & $(-8.53)$ & $(-64.89)$ & $(60.11)$ \\
\hline & \multirow{2}{*}{ Administração Pública } & -0.010 & -0.1209 & 0.1296 \\
\hline & & $(-47.22)$ & $(-64.46)$ & $(93.08)$ \\
\hline & \multirow{2}{*}{ Educação, Saúde e Social } & -0.2275 & -0.2567 & -0.0312 \\
\hline & & $(-99.24)$ & $(-130.59)$ & $(-21.02)$ \\
\hline & \multirow{2}{*}{ Serviços Domésticos } & -0.0168 & -0.0990 & -0.0242 \\
\hline & & $(-17.32)$ & $(-146.52)$ & $(-38.45)$ \\
\hline \multirow{8}{*}{ Região } & \multirow{2}{*}{ Nordeste } & -0.2447 & -0.3167 & -0.2191 \\
\hline & & $(-405.97)$ & $(-670.56)$ & $(-519.29)$ \\
\hline & \multirow{2}{*}{ Sudeste } & 0.0527 & -0.0156 & 0.1083 \\
\hline & & $(95.69)$ & $(-31.04)$ & $(254.12)$ \\
\hline & \multirow{2}{*}{ Sul } & -0.0072 & 0.0064 & 0.1322 \\
\hline & & $(-12.47)$ & $(12.80)$ & $(295.49)$ \\
\hline & \multirow[t]{2}{*}{ Centro-Oeste } & 0.0869 & 0.0631 & 0.2060 \\
\hline & & $(138.17)$ & $(113.04)$ & $(416.22)$ \\
\hline \multirow{7}{*}{ Constante } & & 3.0319 & 3.7262 & 4.6282 \\
\hline & & $(853.80)$ & $(1176.19)$ & $(1971.68)$ \\
\hline & $\mathrm{R} 2$ & 0.3536 & 0.3763 & 0.3450 \\
\hline & Estatística F & 150.0000 & 160.0000 & 130.0000 \\
\hline & Prob> F & 0.0000 & 0.0000 & 0.0000 \\
\hline & Hausman (Prob>chi2) & 0.0000 & 0.0000 & 0.0000 \\
\hline & $\begin{array}{l}\text { No de observações } \\
\text { (amostra extendida) }\end{array}$ & 47188516 & 49162891 & 51795417 \\
\hline
\end{tabular}

Fonte: Cálculo dos autores a partir dos dados da PNAD.

Nota: Estatística $z$ entre parênteses. 
Note-se ainda que a região Nordeste do Brasil possui rendimento médio condicional menor que a renda Norte nos anos de análise. A Região Sul em 2001 também apresentou rendimento menor que a Norte, mas esse rendimento aumentou para os anos 2006 e 2012. A Região Sudeste apresentou rendimento inferior ao Norte no ano de 2006.

\section{Resultados da decomposição}

Lembrando que a decomposição em nível mostra os principais determinantes da desigualdade de renda dos rendimentos no Brasil num determinado período de tempo, enquanto que a decomposição da diferença indica os principais determinantes da variação na desigualdade entre dois períodos distintos.

Para variáveis explicativas que entram na equação de rendimentos como a variável educação, seus respectivos $S_{j}^{\prime} s$ terão uma interpretação direta. No entanto, tal interpretação torna-se diferente para variáveis que entram na equação de formas alternativas, como variáveis, com efeito, não-linear. Para o caso das variáveis como educação, se o seu Sj, ou seja, sua contribuição para explicar a desigualdade é igual a $y \%$, isto significa que tal variável é responsável por $y \%$ da desigualdade num determinado período. Esta interpretação é diferente para variáveis que possuem um efeito não-linear como expe exp2. Neste caso, a contribuição da categoria exppara a desigualdade de renda seria dada pela soma dos seus dois $S_{j}^{\prime} s$ - do linear e do não-linear.

\subsection{Decomposição para o Período 2001-2012}

Pode-se observar na tabela 4, a seguir, que o ano de 2001 apresenta a variável educação como principal determinante da desigualdade de salário. Seu valor, 46.11\%, é superior ao observado em 2012, 32.98\%. Esses resultados estão de acordo comos de Berni (2007) que encontrou que a educação explica 41.82\% da desigualdade de renda em 1995 e 39.12\% em 2005 para o Brasil como um todo. Ramos e Vieira (2001) também encontram um valor de 33\% para o ano de 1999, em nível nacional, o que coincide com o resultado apresentado na nota técnica do IPEA (2006) para o período 2001-2004.

A redução da participação da variável educação na explicação da desigualdade se deve à diminuição dos retornos médios da escolaridade, como se pode ver na tabela 3. Como afirma Soares (2006), o maior acesso à educação pode ter levado a força de trabalho a se tornar mais qualificada e isto tem provocado uma diminuição dos retornos médios da escolaridade num ambiente em que a oferta de trabalho é maior que a demanda.

A segunda contribuição mais importante na explicação da desigualdade é da variável ocupação com 6.66\% seguida por gênero com 5.14\% para 2001.

A última coluna da tabela 4 mostra os resultados da "decomposição da diferença". As 
variáveis educação, gênero, experiência e ocupação são as que contribuem para a diminuição da desigualdade de renda salarial no período 2001-2012. Dentre estas variáveis, a educação é a que apresenta maior importância com 78.46\%.

Tabela 4: Contribuição das variáveis explicativas para a desigualdade de renda. Decomposição em nível e Decomposição da diferença para o Brasil - 2001/2012

\begin{tabular}{c|c|c|c}
\hline $\begin{array}{c}\text { Variáveis } \\
\text { Explicativas }\end{array}$ & $\mathbf{S j}(\mathbf{l n Y}), \mathbf{2 0 0 1}$ & $\mathbf{S j}(\mathbf{l n Y}), \mathbf{2 0 1 2}$ & $\begin{array}{c}\boldsymbol{\Pi}_{\mathbf{j}}(\mathbf{G I N I}), \mathbf{2 0 0 1}- \\
\mathbf{2 0 1 2}\end{array}$ \\
\hline Educação & 0.4611 & 0.3298 & 0,7846 \\
\hline Gênero & 0.0514 & 0.0493 & 0,0565 \\
\hline Raça & 0.0034 & 0,0060 & $-0,0030$ \\
\hline Experiência & -0.0123 & -0.0221 & 0,0121 \\
\hline Sindicato & 0.0005 & 0.001 & $-0,0003$ \\
\hline Ocupação & 0.0666 & 0.0794 & 0,0349 \\
\hline Atividade & -0.0055 & 0.0160 & $-0,0584$ \\
\hline Resíduo & 0.4348 & 0.5408 & 0,1736 \\
\hline
\end{tabular}

Fonte: Elaborado pelos autores.

\subsection{Decomposição para o Período 2006-2012}

Os resultados na tabela 5, a seguir, indicam que a variável educação é o principal determinante da desigualdade de salários do Brasil. Verifica-se que para os anos de 2006 e 2012, os anos de escolaridade das pessoas ocupadas explicam, respectivamente, 41.61\% e $32.98 \%$, da desigualdade.

A variável ocupação é o segundo determinante mais importante da desigualdade salarial em 2006, com uma contribuição de 9.64\%. No entanto, este valor passa para apenas 7.94\% em 2012.

Em seguida, na ordem de importância, aparece a variáveigênero com 5.14\% em 2006 e 4.93\% em 2012, influenciado pela diminuição na discriminação salarial entre homens e mulheres.

Vale observar que o resíduo na "decomposição em nível” é de aproximadamente 50\%. Este resíduo mostra que as variáveis explicativas da equação de salários não explicam mais que a metade da desigualdade de salários. Estes resíduos, apesar de elevados, também são observados nos trabalhos de Fields (2002) e Contreras (2003). Berni (2007), encontra para os períodos 1995, 2001 e 2005, os resíduos de 0.46, 0.40 e 0.49, respectivamente. 
180 - Desigualdade de renda salarial no Brasil no período 2001 a 2012

Tabela 5: Contribuição das variáveis explicativas para a desigualdade de renda

Decomposição em nível e Decomposição da diferença para o Brasil - 2006/2012

\begin{tabular}{c|c|c|c}
\hline Variáveis Explicativas & $\mathbf{S j}(\mathbf{l n Y}), \mathbf{2 0 0 6}$ & $\mathbf{S j}(\mathbf{l n Y}), \mathbf{2 0 1 2}$ & $\begin{array}{c}\boldsymbol{\Pi}_{\mathbf{j}}(\mathbf{G I N I}) \mathbf{2 0 0 6}- \\
\mathbf{2 0 1 2}\end{array}$ \\
\hline Educação & 0.4161 & 0.3298 & 0.7687 \\
\hline Gênero & 0.0514 & 0.0493 & 0.0600 \\
\hline Raça & 0.0020 & 0,0060 & -0.0143 \\
\hline Experiência & -0.0140 & -0.0221 & 0.0190 \\
\hline Sindicato & 0.00085 & 0.001 & 0.0012 \\
\hline Ocupação & 0.0964 & 0.0794 & 0.1656 \\
\hline Atividade & -0.0101 & 0.0160 & -0.1168 \\
\hline Resíduo & 0.4573 & 0.5408 & 0.1166 \\
\hline
\end{tabular}

Fonte: Elaborado pelos autores.

Pode-se perceber, na última coluna da tabela 5, que as variáveis educação, gênero, experiência,sindicato e ocupação são as que contribuem para a diminuição da desigualdade de renda salarial no período 2006-2012. Dentre estas variáveis, a educação é a que apresenta maior importância com 76.87\%. As variáveis com sinal negativo não contribuíram para a diminuição da desigualdade.

\section{Considerações finais}

Este trabalho utilizou uma metodologia de decomposição - definida em Fields (2002) - da desigualdade de renda dos salários no Brasil capaz de indicar quais são as principais variáveis que contribuíram para a explicação da desigualdade de renda. Inicialmente estimouse a equação de rendimentos e em seguida, aplicou-se a decomposição em nível e em diferença. Antes de realizar a decomposição foi possível mostra que a desigualdade de renda no Brasil vem caindo nos últimos anos por meio da determinação do coeficiente de Gini. Entretanto, verifica que essa desigualdade ainda é muito elevada.

Os resultados obtidos para os coeficientes da variável educação, os retornos médios da escolaridade foram de 22.32\%, 20.66\% e 16.66\% para os anos 2001, 2006 e 2012, respectivamente. Com isso, já se pode notar que, apesar de ser a variável mais importante para explicar o salário, a educação vem apresentando diminuição de seu retorno médio.

Para a variável raça, verifica-se que existe discriminação de cor. Por exemplo, no ano de 2001 os brancos ganhavam em média 2.51\% a mais que as outras raças. Esta discriminação segue em queda durante o período analisado, pois em 2006 os brancos recebiam em média $1.56 \%$ a mais que pessoas de outras raças. Já em 2012, esse percentual aumentou, os brancos recebiam em média, 4.57\% a mais que os negros, 
indígenas, parda, e amarela. Logo, as outras raças possuem em média rendimentos inferiores aos brancos.

A variável educação mostrou ser a mais importante na explicação da desigualdade para o ano de 2001 na "decomposição em nível", seguida da variável ocupação com 6.66\% e Gênero com 5.14\% para 2001. Em 2006, a variável educação também foi a mais importante, seguida a variável ocupação é a segunda determinante mais importante da desigualdade salarial em 2006, com uma contribuição de 9.64\%.Para o ano de 2012, a educação continuou como a principal determinante da desigualdade de renda, também seguida pela variável ocupação e gênero.

Com relação à "decomposição da diferença", a variável educação mostrou-se também a mais importante, tanto no período 2001-2012, quanto no período 2006-2012, embora seu peso tenha sido maior no segundo caso. A variável gênero ocupa o segundo lugar neste tipo de decomposição para os dois períodos.

Em linhas gerais, foi constatado que pela estimação da equação de salários e pelo método de decomposição em nível utilizado, a educação mostrou ser o fator mais importante na explicação da desigualdade para o período os anos de 2001,2006 e 2012. Concluísse que modificações nos níveis educacionais da população brasileira, possuem uma importância considerável na desigualdade de renda, o que deve ser considerado como um fator relevante na consecução para reduzir a desigualdade de renda.

\section{Referências bibliográficas}

ARAÚJO, J. A.; FEITOSA, D. G.; BARRETO, F. A. D. F. Determinantes da desigualdade de renda em áreas rurais do Nordeste. Revista de Política Agrícola, v. 17. n. 4, p.65-82, 2008.

BARROS, R. P.; MENDONÇA, R. S. P. Determinantes da Desigualdade no Brasil. IPEA. Textos para Discussão n. 337, 1995.

.Os determinantes da desigualdade no Brasil. Programa de seminários acadêmicos do IPE/USP. Seminário número 22/97, out. 1997.

BARROS, R. P.; CARVAlHO, M. de; FRANCO, S.; MEDONÇA, R. Determinantes Imediatos da Queda da Desigualdade Brasileira. Rio de Janeiro: IPEA, jan. 2007. (Texto para Discussão, $\mathrm{N}^{\circ} 1253$ ).

BARROS,R.P.; FOGUEL, M.N.; ULYSSEA, G. Desigualdade de renda no Brasil: uma análise da queda recente. $1^{\text {a }}$ ed. Brasília: Ipea, 2007.

BERNI, H. A. de A. Evolução dos Determinantes da Desigualdade de Renda Salarial no Nordeste. 2007. 48f. Dissertação (Mestrado em Economia) - CAEN, Universidade Federal do Ceará, Fortaleza, 2007. 
CAMPANTE, F. R.; CRESPO, A. V.; LEITE, P. Desigualdade Salarial entre Raças no Mercado de Trabalho Urbano Brasileiro: Aspectos Regionais. Rio de Janeiro: Revista Brasileira de Economia, vol. 58 abril/jun, p. 186-209, 2004.

CARD, D. Using Geographic Variation in College Proximity to Estimate the Return to Schooling. NBER. Discussion Paper n. 4483. 1993.

CONTRERAS, D. Poverty and inequality in a rapid growth economy: Chile 1990- 1996. The Journal of Development Studies, v.39, p.181-200, fev.2003.

CRUZ, M. S. da; BARRETO, F. A.; SANTOS, L. M. dos; SOUTO, K. C. de. Determinantes das Desigualdades Salariais nas Macrorregiões Brasileiras: Uma Análise para o Período 2001 - 2006. Revista Econômica do Nordeste, Fortaleza, v. 42, n. 3, p. 551-575, jul-set., 2011.

DE HOYOS, R. E. Accounting for Mexican Income Inequality During the 1990's. México: World Bank. 2006.

DE LA CROIX, D.; DOEPKE, M. Inequality and Growth: Why Differential Fertility Matters. The American Economic Review. Vol 93. n. 4. p.1093-1113, 2003.

FIELDS, G. S. Accounting for income inequality and its changes: A new method with application to the distribution of earnings in the United States.DepartmentofEconomics. Cornell University. Working Paper.v.22, p.139-60. 2002.

INSTITUTO DE PESQUISA ECONÔMICA APLICADA - IPEA. Sobre a Recente Queda da Desigualdade de Renda no Brasil. Nota Técnica. Agosto, 2006.

INSTITUTO DE PESQUISA ECONÔMICA APLICADA- IPEA. Sobre "A Década Inclusiva (2001-2011): Desigualdade, Pobreza e Políticas de Renda”. 2012.

KAKWANI, N.; NERI, M.; SON, H. Linkages between pro-poor growth, social programmes and labour market: the recent Brazilian experience. WorkingPaper, Brasil: PNUD, 2006.

HOFFMANN, R. A distribuição de renda no Brasil no período 1992-2001. Economia e Sociedade, Campinas, v.11, 2002.

.Transferência de renda e a redução da desigualdade no Brasil e cinco regiões entre 1997 e 2004. Econômica, v. 8, n. 1, p. 55-81, jun. 2006. Disponível em: $<$ http://www.uff.br/cpgeconomia/economica.htm>, acesso em 16 out. 2013.

LAM, D.; LEVINSON, D. Declining inequality of schooling in Brazil and its effect on inequality of wages. Journal of Development Economics, v. 37. p. 199-225, 1992. 
LAM, D. Generating Extreme Inequality: Schooling. Earnings, and Intergenerational Transmission of Human Capital in South Africa and Brazil.Universityof Michigan, processed. 1999.

LANGONI, G. Distribuição de Renda e Crescimento Econômico, Rio de Janeiro: Expressão e Cultura. 1973.

MARIANO, F. Z.; ARRAES, R. D. A. Endogeneidade da educação na previsão da taxa de retorno: avaliação metodológica e aplicação para regiões brasileiras e estados selecionados. IPECE, Fortaleza, 2011. Disponível em < http://www.ipece.ce.gov.br>, acesso em 16 out. 2013.

OLIVEIRA, R. C.; SILVEIRA, R. M. Escolaridade, políticas sociais e a evolução da desigualdade Regional de renda no Brasil entre 2003 e 2011: uma análise a partir das fontes de renda. Fórum BNB, 2013.

PINHO NETO, V. R.; BARRETO, F. A. F. D.; FEIJÓ, J. R. A Importância da Educação para a Recente Queda da Desigualdade de Renda Salarial no Brasil: Uma análise de decomposição para as regiões Nordeste e Sudeste.IPEA, 2011.

PSACHAROPOULOS, G. Time Trends of the Returns to Education: Cross- National Evidence. Economics and Education Review, n. 8, v. 3, 1991.

RAMOS, L.; VIEIRA, M. L. Determinantes da Desigualdade de Rendimentos no Brasil nos Anos Noventa: Discriminação, Segmentação e Heterogeneidade dos Trabalhadores. IPEA. Texto para Discussão, n. 803, 2001.

RESENDE, M.; WYLLIE, R. Retorno para Educação no Brasil: Evidências Empíricas Adicionais. Texto para discussão n.03, UFRJ, Rio de Janeiro. 2005.

SAlVATO, M. A.; DUARTE, A. J. M. A; FERREIRA, P. C. Disparidades Regionais ou Educacionais? Um exercíciocontrafactual.Mimeografado, Jul, 2003.

SALVATO, M. A.; LIMA, L. F.; VIANA, J. S. Accounting for Income Inequality: An Application of the Fields Methodology to the Recent Fall of Inequality in Brazil.In: ENCONTRO NACIONAL DE ECONOMIA DA ANPEC. 2013, Fortaleza, CE. Anais... Fortaleza: ANPEC, 2013.

SACHSIDA, A.; LOUREIRO, P. R. A.; MENDONÇA, M. J. C. Um Estudo Sobre Retorno em Escolaridade no Brasil. RBE. Rio de Janeiro: Abril/Jun, 2004.

SOARES, S. D. S. Análise de bem-estar e decomposição por fatores da queda na desigualdade entre 1995 e 2004. Econômica, Rio de Janeiro, v.8, n.1, p.83-115, jun. 2006. 
184 - Desigualdade de renda salarial no Brasil no período 2001 a 2012

SIQUEIRA, M. L.; SIQUEIRA, M. L. Desigualdade de Renda no Nordeste Brasileiro: Uma Análise de Decomposição. XI Encontro Regional de Economia, ANPEC, Banco do Nordeste. Maio, 2006.

SHORROCKS, A. F. Inequality Decomposition by Factor Components. Econometrica,v. 50, n.1, p. 193-211, 1982.

SQUIRE, L.; ZOU, H. Explaining International and Intertemporal Variations in Income Inequality. The Economic Journal, v. 108. p.26-43, 1998.

STOCK, H. J.; WATSON, W. M. Econometria. Pearson, Addison Wesley. 2004.

SUliANO, D. C.; SIQUEIRA, M. L. Um Estudo do Retorno da Educação na Região Nordeste: Análise dos Estados da Bahia, Ceará e Pernambuco a partir da Recente Queda da Desigualdade. IPECE,Texto para discussão n 72, 2010. Disponível em <http://www.ipece.ce.gov.br/publicacoes/textos_discussao/TD_72.pdf>, acesso em 8 out. 2013.

WOOLDRIDGE, J. M. Econometric Analysis of Cross Section and Panel Data.The MIT Press, Cambridge, MA. 2002. 